\title{
A NOTE ON THE CONSTRUCTION OF A SET OF
}

\section{FUNDAMENTAL CIRCUITS ON A SURFACE OF GENUS $P$}

\author{
R. G. de Buda
}

(received September 26, 1960)

On an orientable surface of genus $p$, a set of $2 p$ fundamentalcircuits can be selected which all pass through a single point A. After cutting along the 2 p circuits, the surface can be unfolded into a flat region bounded by a $4 \mathrm{p}$-gon so that: the set of vertices corresponds to the one point $A$; and the $2 \mathrm{p}$ pairs of edges to the $2 \mathrm{p}$ fundamental circuits; and the interior of the polygor to the remainder of the surface. If the edges of the polygon are directed, the 2 edges which correspond to one fundamental circuit will be directed in opposite sense, since the surface is orientable [1]. The sequence and direction of the edges is the same as the sequence of the fundamental circuits.

Conversely, if a $4 \mathrm{p}$-gon is given with a prescribed sequence of its directed edges, it may be required to construct a system of $2 p$ fundamental circuits on a surface of genus $p$, so that (after cutting) the circuits can be identified with the edges of the $4 \mathrm{p}$-gon in their prescribed sequence. Of course, such a construction will only be possible if the given $4 \mathrm{p}$-gon does correspond to a surface of genus $p$; for this the following two conditions must be fulfilled by the 4 p-gon:

1) Each fundamental circuit corresponds to two edges of the polygon, and these two edges must be directed in opposite sense. This is of course, the condition of orientability of the surface.

2) The $4 p$ vertices of the polygon form one and only one conjugate set [1]. Consequently all vertices correspond to one point of the mapped surface, and by one continuous contour which crosses all edges it is possible to enclose all $4 p$ vertices.

Canad. Math. Bull. vol. 4, no. 1, January 1961 
This is the condition that the surface be of genus $p$, because the fundamental circuits intersect in a single point, only if there is no more than one conjugate set of vertices; and counting 1 face, $2 \mathrm{p}$ pairs of edges, and 1 conjugate set of vertices, the Euler characteristic becomes [2] $\chi=N_{0}-N_{1}+N_{2}$ $=2-2 p$ which is the characteristic of a surface of genus $p$. But, if the vertices form more than one conjugate set, $\mathrm{N}_{0}>1$, then $\chi>2-2 p$ and the $4 p$-gon represents a surface of genus less than $p$.

The number of conjugate sets of vertices can be obtained by drawing closed contours around the vertices of the $4 \mathrm{p}$-gon, and counting the conjugate sets (each of which is enclosed by a different contour). In order to draw a contour a round a conjugate set of vertices, corresponding edges should be easily identified on the sketch. For this it is of advantage to map the surface not on the inside but on the outside of the $4 \mathrm{p}-\mathrm{gon}$. Topologically there is of course no difference, provided the $4 \mathrm{p}$-gon is regarded as having been drawn on a sphere or in the inversive plane, but it is convenient to have the inside available to draw a continuation of the contour between corresponding edges. Figs.1a and $1 \mathrm{~b}$ show 2 polygonal normal forms of the surface of genus 2 , with all 8 vertices arranged in one conjugate set, so that one circuit encloses all vertices. The corresponding surface would be a sphere with 2 handles or a solid figure of eight [3]. Figs.1c and 1d show two octagons, which correspond to a surface of genus 1 only, since in both cases the vertices divide into 3 conjugate sets, as shown by the 3 distinct contours. Sketches similar to figs. $1 \mathrm{a}-\mathrm{d}$ can easily be constructed to test whether a given polygon with $2 \mathrm{p}$ pairs of directed edges does or does not form a map of an orientable surface of genus $p$.

In particular, when the polygon is in the second canonical form [4],

$$
a_{1} a_{2} \cdots a_{2 p} a_{1}^{-1} a_{2}^{-1} \cdots a_{2 p}^{-1}
$$

a sketch similar to fig. $1 \mathrm{~b}$ shows that a contour a round the vertices consists of arcs around the vertices (as identified by their adjacent edges) in the following sequence: 


$$
a_{1} a_{2} ; a_{2}^{-1} a_{3}^{-1} ; a_{3} a_{4} ; \ldots . ; a_{2 p-1} a_{2 p} ; a_{2 p}^{-1} a_{1} ; a_{1}^{-1} a_{2}^{-1} \text { etc. }
$$

This contour therefore encloses all $4 \mathrm{p}$ vertices so that the vertices form one conjugate set, and the polygon forms a surface of genus $p$.

Consider now the surface to be mapped on the interior of a polygon which has 2 p pairs of directed edges and fulfils conditions 1 and 2. Take a point $B$ in the interior of the polygon in which intersect $2 \mathrm{p}$ undirected lines, connecting corresponding edges. Taken on the original surface, these lines cut the interior of the $4 \mathrm{p}$-gon into $4 \mathrm{p}$ sectors, and each such sector connects to one vertex of the $4 \mathrm{p}$-gon. If all vertices form one conjugate set, so that one contour encloses them all, the vicinities of the vertices must be connected. Since each sector connects to one vertex, the region resulting from cutting the original surface along the new system of circuits must be still a connected region. But since the region is obtained by cutting $2 p$ times a surface of genus $p$, it follows that the region resulting from the new cuts, if connected, is simply connected [5]; therefore the new circuits are a new set of $2 \mathrm{p}$ fundamental circuits. (Incidentally this shows again that this surface cannot be of a genus less than p.) Consequently, as illustrated in fig. 2, if a polygonal normal form representing a surface of genus $p$ is given, a set of fundamental circuits can be obtained as a system of $2 \mathrm{p}$ circuits on the surface which intersect in one point $B$ only and their sequence of intersection (sequence in which they cross a contour around their point of intersection) is the same sequence in which the directed edges of the $4 \mathrm{p}$-gon follow each other. The resulting region can be unfolded into a flat region bounded by a second $4 \mathrm{p}$-gon, the $4 \mathrm{p}$ vertices of which form one conjugate set corresponding to point $B$; while point $A$ is now in the interior, as well as all fundamental circuits of the first set (which intersect in A). The two 4 p-gons and the two sets of $2 \mathrm{p}$ fundamental circuits stand in dual relation to each other [6], and the circuits of one set follow in the same sequence in which the circuits of the other set intersect. In other words, we have, on the surface of genus $p$, two dual maps, each having one vertex and one face.

Therefore, $2 p$ circuits, which intersect only in one point, and in the same sequence in which the contour a round the single conjugate set of vertices of the given 4 -gon crosses its edges, 
will be the fundamental set with the sequence prescribed by the edges of this $4 \mathrm{p}$-gon.

It will usually be possible to find such circuits by inspection, routing them so that they do not cross each other a second time. Fig. 3 illustrates a set of fundamental circuits which follow in the sequence $a_{1} a_{2} \cdot a_{2 p} a_{1}^{-1} \ldots a_{2 p}^{-1}$ and intersect in the sequence $a_{1} a_{2}^{-1} a_{3} a_{4}^{-1} \ldots a_{2 p}^{-1} a_{1}^{-1} a_{2} a_{3}^{-1} \cdots a_{2 p}$.

It remains to show that for any orientable surface of genus $p$, as represented by a sphere with $p$ handles, such a system of cuts can be constructed in a systematic way. Consider two adjacent edges $x, y$ in the polygon, with edges arranged $x y P x^{-1} Q y^{-1} R$ (whe re $P, Q, R$ stand for blocks of otherwise unspecified edges). Then the 2 circuits which cross edges $x$ and $y$ intersect. Such a pair of edges $x, y$ must always exist, lotherwise there must be a circuit which encloses only one vertex, so that the polygon cannot represent a surface of genus p). These two circuits can be arranged in such a way as to cut open one handle in a manner which is illustrated in fig. 4. All other circuits are brought out and into the vicinity of the next handle along the ducts shaded in fig. 4. At this point another pair of circuits is selected which intersect. Such a pair must exist, or the surface remaining after removal of the first handle could not be of genus $p-1$, and this would lead to a contradiction. These 2 circuits will cut the next handle open.

The process can be continued until all $\mathrm{p}$ handles are cut along the system of circuits thus obtained. The fundamental circuits can then be drawn by $p$ times repeating fig. 4 . The "routing" of the circuits around the handles which they do not remove, corresponds closely to the substitutions made to move the blocks of edges [7] to bring edges $x$ and $y$ into the sequence $x y x^{-1} y^{-1} Q$ during the construction of the first canonical form.

\section{REFERENCES}

1. H. R. Brahana, Systems of circuits on two dimensional manifolds, Ann. of Math. 23(1921), 144-168. 
2. H. S. M. Coxeter and W. O.J. Moser, Generators and relations for discrete groups, Ergebnisse der Mathematik 14, (Berlin,1957), equation 3.55.

3. W. Burnside, Theory of groups of iinite order, (London, 1911), fig. 8 , p. 396.

4. W. Threlfall, Gruppenbilder, Abh. Sächs. Akad. Wiss. Math. - Phys. KI. 41, no. 6, 1-59, particularly p. 35.

5. D. Hilbert and S. Cohn Vossen, Geometry and the Imagination, second edition, (New York, 1952), p. 296 and figures on pp. 300-301.

6. J. Nielsen, Untersuchungen zur Topologie der geschlossenen zweiseitigen Flaechen, Acta Math. 50 (1927) 189-358, particularly p. 195.

7. R. C. James, Combinatorial topology of surfaces, Math. Mag. 29 (1955), 1-39.

Canadian General Electric Co. Ltd. , Toronto, Ont. 
FIG. I

POLYGONS AND CONTOURS AROUND THE CONJUGATE SETS OF VERTICES.

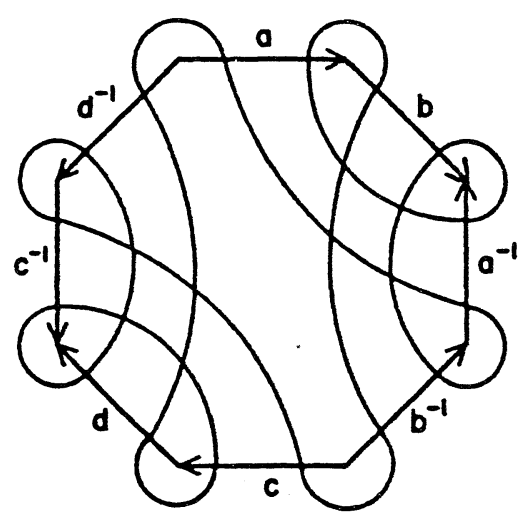

(a)

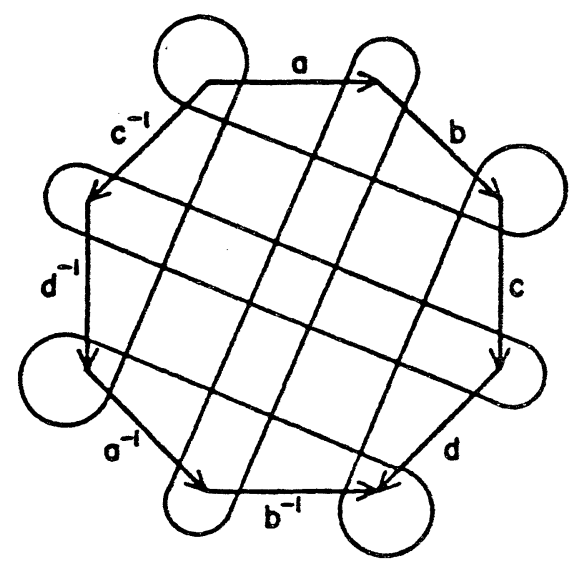

(c)

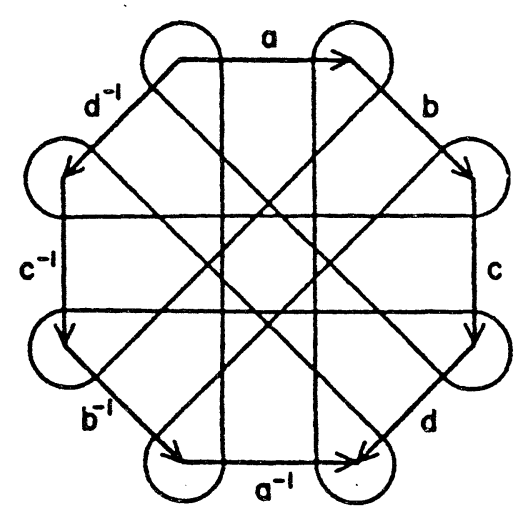

(b)

$D, b$ ONE CONTOUR; GENUS $p=2$

c, $d$ CONTOURS; GENUS $p=1$

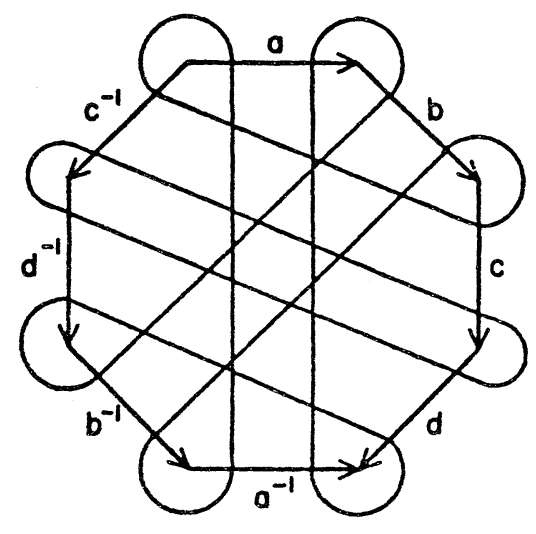

(d) 
FIG. 2

SEQUENCE OF INTERSECTION OF FUNDAMENTAL CIRCUITS.

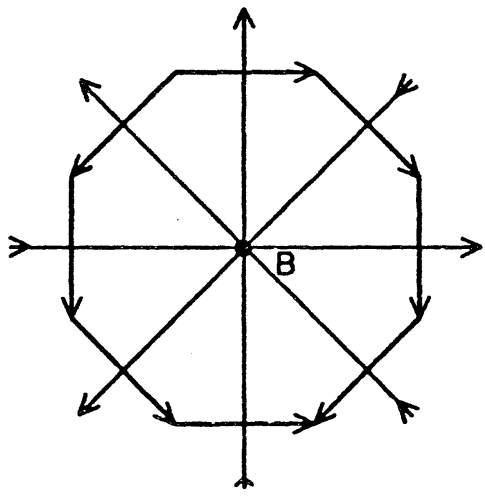

(a)

$$
a b^{-1} c d^{-1} a^{-1} b c^{-1} d
$$

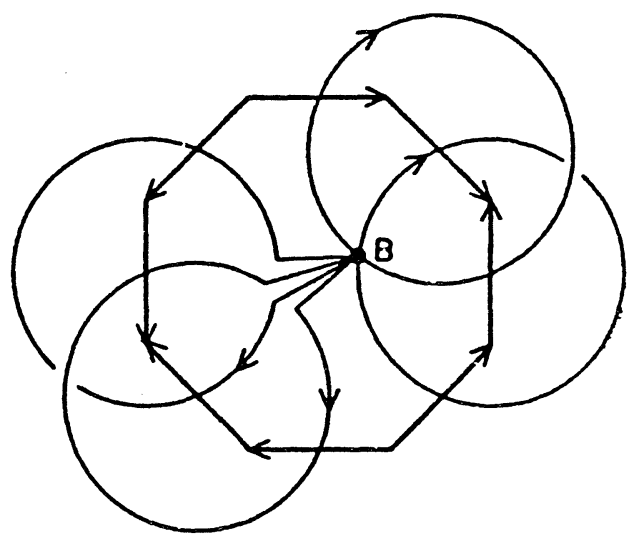

(b) $a b a^{-1} b^{-1} c d c^{-1} d^{-1}$ 
FIG. 3
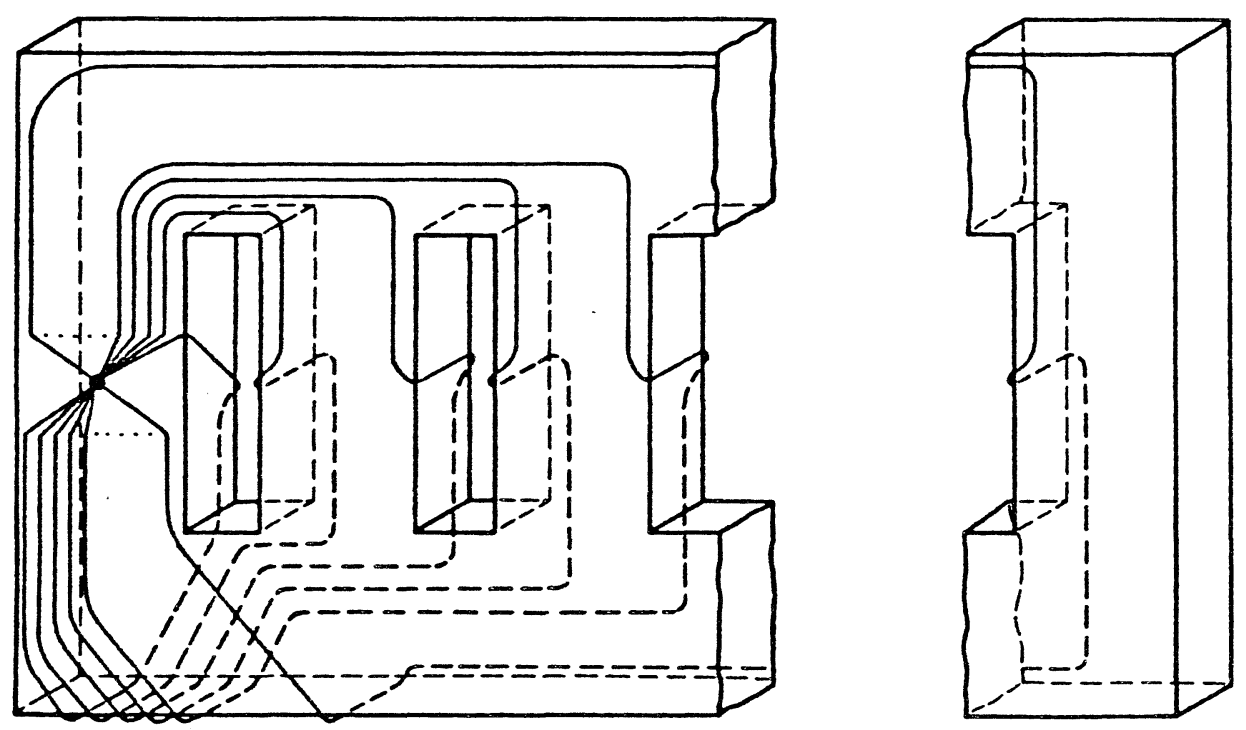

SET OF FUNDAMENTAL CIRCUITS FOR $a_{1} a_{2} \ldots a_{2 p} a_{1}^{-1} \ldots \ldots a_{2 p}^{-1}$ 
FIG. 4

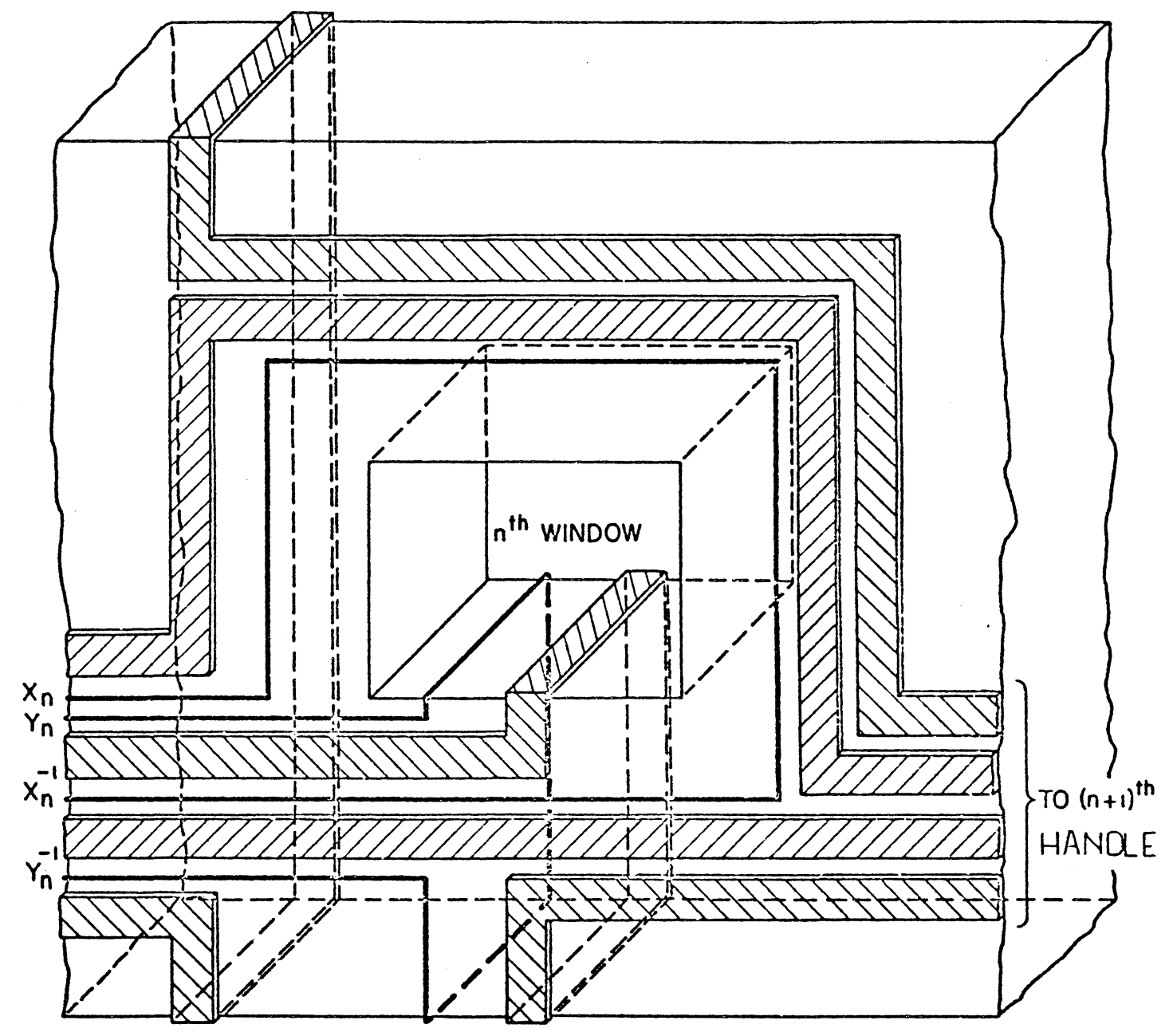

REMOVAL OF THE $n^{\text {th }}$ HANDLE BY CIRCUITS $x_{n}$ AND $Y_{n}$ THE OTHER CIRCUITS ARE ROUTED ALONG THE CHANNELS SHOWN SHADED. 